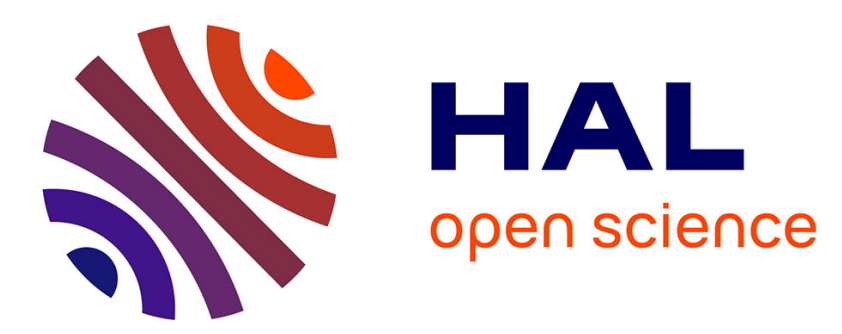

\title{
Selective control of Poiseuille-Rayleigh-Bénard instabilities by a spanwise magnetic field
}

\author{
Walid Fakhfakh, Slim Kaddeche, Daniel Henry, Hamda Ben Hadid
}

\section{To cite this version:}

Walid Fakhfakh, Slim Kaddeche, Daniel Henry, Hamda Ben Hadid. Selective control of PoiseuilleRayleigh-Bénard instabilities by a spanwise magnetic field. Physics of Fluids, 2010, 22, pp.034103. 10.1063/1.3327287 . hal-00566033

\section{HAL Id: hal-00566033 https://hal.science/hal-00566033}

Submitted on 7 Jun 2012

HAL is a multi-disciplinary open access archive for the deposit and dissemination of scientific research documents, whether they are published or not. The documents may come from teaching and research institutions in France or abroad, or from public or private research centers.
L'archive ouverte pluridisciplinaire HAL, est destinée au dépôt et à la diffusion de documents scientifiques de niveau recherche, publiés ou non, émanant des établissements d'enseignement et de recherche français ou étrangers, des laboratoires publics ou privés. 


\title{
Selective control of Poiseuille-Rayleigh-Bénard instabilities by a spanwise magnetic field
}

\author{
W. Fakhfakh, ${ }^{1}$ S. Kaddeche, ${ }^{1}$ D. Henry, ${ }^{2}$ and H. Ben Hadid ${ }^{2}$ \\ ${ }^{1}$ Unité de Recherche Matériaux, Mesures et Applications, \\ Institut National des Sciences Appliquées et de Technologie (INSAT), B.P. 676, 1080 Tunis Cedex, Tunisia \\ ${ }^{2}$ Laboratoire de Mécanique des Fluides et d'Acoustique, CNRS/Université de Lyon, \\ Ecole Centrale de Lyon/Université Lyon 1/INSA de Lyon, ECL, 36 Avenue Guy de Collongue, \\ 69134 Ecully Cedex, France
}

(Received 10 June 2009; accepted 14 January 2010; published online 18 March 2010)

\begin{abstract}
The stability of a pressure driven flow in an electrically conducting fluid heated from below and subjected to a spanwise constant magnetic field is investigated through a linear stability analysis. The numerical calculations show that such a magnetic field only affects the longitudinal stationary modes $(L)$, which are stabilized, and has no effect on the transverse traveling modes $(T)$. A direct consequence is the expansion of the domain where the transverse traveling $(T)$ modes prevail. This expansion is controlled by the strength of the spanwise magnetic field, expressed through the Hartmann number (Ha, ratio of magnetic to viscous dissipation forces). Moreover, when Ha exceeds a limiting value depending on the Prandtl number, the $(T)$ modes become the only dominant modes in the whole Re range. Particular attention was dedicated to the oblique modes $(O)$ corresponding to fully three-dimensional disturbances in order to verify that they never become the dominant modes when the magnetic field is applied, similarly to what was found without magnetic field. From a practical point of view, these results could be of a great interest since it is known that the optimization of several processes involving the Poiseuille-Rayleigh-Bénard flow is achieved when the traveling (T) modes prevail. (C) 2010 American Institute of Physics. [doi:10.1063/1.3327287]
\end{abstract}

\section{INTRODUCTION}

Among the thermoconvective flows, the PoiseuilleRayleigh-Bénard (PRB) configuration is a particularly interesting system from both fundamental and practical points of view. Such flow is involved in many industrial and earthscience problems. A crucial aspect of the PRB flow is its transition toward instability. The investigation of the instability problem appears to be significantly important because the instabilities of the PRB flows directly affect various technological and engineering problems such as chemical vapor deposition $(\mathrm{CVD})^{1,2}$ and the cooling of electronic devices. ${ }^{3-6}$ Generally, in these processes, the traveling transverse modes $(T)$ are preferred to the stationary longitudinal modes $(L)$. In CVD applications, the $(L)$ modes are responsible for the formation of undesirable striations parallel to the channel axis. $^{7-10}$ Furthermore, the stationary character of the $(L)$ modes does not allow the optimization of the heat transfer during the cooling of electronic equipments. For the improvement of mass and heat transfer in such processes, it is then necessary to find out a way to control these instabilities and promote the transverse traveling $(T)$ modes in the range of the operating parameters.

To our knowledge, in the framework of magnetohydrodynamics, only Fakhfakh et al. ${ }^{11}$ have considered the effects of a magnetic field on the instabilities occurring in the PRB system. For $\operatorname{Pr}=0.001$, it has been shown that a vertical magnetic field stabilizes both $(T)$ and $(L)$ modes. The authors ${ }^{11}$ also showed that the critical Reynolds number for the dominantly growing $(T)$ modes increases with increasing Hartmann number and is an order of magnitude higher than the operating Reynolds number for $\mathrm{CVD}^{12,13}$ and the cooling of electronic devices. ${ }^{14,15}$ This results in a narrower range of Reynolds numbers where the $(T)$ modes dominate. As an example, Fakhfakh et al. ${ }^{11}$ showed that the traveling $(T)$ modes prevail from $\mathrm{Re}=5490$ to $\mathrm{Re}=5772$, i.e., in a $\operatorname{Re}$ range $\Delta \mathrm{Re}=282$, for $\mathrm{Ha}=0$ (case without magnetic field) and from $R e=43962$ to $R e=43975$, i.e., in a $R e$ range $\Delta R e=13$, for $\mathrm{Ha}=2.5$. These results clearly indicate that the vertical magnetic field is not the appropriate solution to make the $(T)$ modes dominate, especially for experimental and industrial situations generally characterized by moderate values of the Reynolds number.

Kaddeche et al. ${ }^{16}$ showed that a magnetic field, either parallel or perpendicular to the basic flow, allows a selective stabilization of the transverse and the longitudinal modes occurring in the Hadley flow (flow induced by a horizontal temperature gradient). For these particular horizontal orientations, their results highlight that the magnetic field has no effect on the instabilities when its direction is parallel to the marginal cell axis and has an optimal stabilizing effect when its direction is perpendicular to the marginal cell axis. Thus, the use of a spanwise magnetic field [which would only stabilize the $(L)$ modes] could be a way to promote the traveling $(T)$ modes in the PRB system. This is what we want to study in this paper. We will investigate the effect of a constant spanwise magnetic field on both $(T)$ and $(L)$ modes which appear in a PRB flow in an infinite layer of an electrically conducting fluid. A detailed description of the domains where such $(T)$ or $(L)$ modes are dominant will be given. The case of oblique modes (corresponding to fully three- 
dimensional disturbances) will be considered in order to check that they are never dominant. Finally, an energy analysis will be performed to get a deeper physical understanding of the instability mechanisms.

\section{PROBLEM FORMULATION}

\section{A. Governing equations and basic flow}

We consider an extended fluid layer of height $H$ confined between two horizontal rigid plates. A constant pressure gradient acting in the $x$ direction drives a plane Poiseuille flow between the plates. This flow is heated from below by imposing a positive temperature difference $\Delta \bar{T}$ between the upper plate maintained at the temperature $\bar{T}_{u}$ and the lower plate maintained at $\bar{T}_{l}$. The fluid is assumed to be Newtonian with constant kinematic viscosity $\nu$ and thermal diffusivity $\kappa$. According to the Boussinesq approximation, the fluid density is considered as constant, except in the buoyancy term where it depends linearly on temperature, $\rho=\rho_{m}\left[1-\beta\left(\bar{T}-\bar{T}_{m}\right)\right]$, where $\beta$ is the thermal expansion coefficient, $\bar{T}_{m}$ is the mean temperature, $\bar{T}_{m}=\left(\bar{T}_{u}+\bar{T}_{l}\right) / 2$, and $\rho_{m}$ is the value of the density at $\bar{T}_{m}$. The fluid is electrically conducting with a constant electric conductivity $\sigma_{e}$ and subject to an external constant magnetic field, $\vec{B}=B \overrightarrow{e_{B}}$. Moreau ${ }^{17}$ showed that in most laboratory experiments using molten metals, the induced magnetic field is negligible so that the applied magnetic field can be considered as the effective magnetic field. Considering $H / 2, U_{\max }, H / 2 U_{\max }, \rho_{m} U_{\max }^{2}, \Delta \bar{T}$, and $B U_{\max } H / 2\left(U_{\max }\right.$ is the velocity at midheight in the channel) as scale quantities for length, velocity, time, pressure, temperature, and electric potential, respectively, the dimensionless governing equations can be written as

$$
\begin{aligned}
& \vec{\nabla} \cdot \vec{V}=0 \\
& \frac{\partial \vec{V}}{\partial t}+(\vec{V} \cdot \vec{\nabla}) \vec{V}=-\vec{\nabla} P+\frac{1}{\operatorname{Re}} \nabla^{2} \vec{V}+\frac{\mathrm{Ra}}{8 \operatorname{Re}^{2} \operatorname{Pr}} T \overrightarrow{e_{z}}+\frac{\mathrm{Ha}^{2}}{\operatorname{Re}} \vec{J} \\
& \times \overrightarrow{e_{B}} \\
& \frac{\partial T}{\partial t}+(\vec{V} \cdot \vec{\nabla}) T=\frac{1}{\operatorname{RePr}} \nabla^{2} T \\
& \vec{\nabla} \cdot \vec{J}=0
\end{aligned}
$$

where the dimensionless variables are the velocity vector $\vec{V}=(U, V, W)$, the pressure $P$, the temperature $T$ [defined as $\left.T=\left(\bar{T}-\bar{T}_{u}\right) / \Delta \bar{T}\right]$, and the induced electric current density $\vec{J}$. The nondimensional parameters arising from the scaling of the equations are the Reynolds number $\operatorname{Re}\left(\operatorname{Re}=H U_{\max } / 2 \nu\right)$, the Rayleigh number $\operatorname{Ra}\left(\operatorname{Ra}=g \beta \Delta \bar{T} H^{3} / \nu \kappa\right)$, the Prandtl number $\operatorname{Pr}(\operatorname{Pr}=\nu / \kappa)$, and the Hartmann number $\mathrm{Ha}$ $\left[\mathrm{Ha}=B(H / 2) \sqrt{\sigma_{e} / \rho_{m} \nu}\right]$. The dimensionless electric current density $\vec{J}$ appearing in the Lorentz force $\left(\mathrm{Ha}^{2} / \mathrm{Re}\right) \vec{J} \times \overrightarrow{e_{B}}$ in Eq. (2) is given by Ohm's law for a moving fluid,

$$
\vec{J}=-\vec{\nabla} \Phi+\vec{V} \times \overrightarrow{e_{B}}
$$

where $\Phi$ is the dimensionless electric potential. Combining the continuity equation for $\vec{J}$ [Eq. (4)] and Ohm's law [Eq. (5)], we obtain the dimensionless equation governing the electric potential $\Phi$,

$$
\nabla^{2} \Phi=\overrightarrow{e_{B}} \cdot(\vec{\nabla} \times \vec{V}) .
$$

The nondimensional basic steady state in the extended layer can easily be obtained without magnetic field. It corresponds to a parabolic $z$ profile for the $x$ component of the velocity vector [throughflow $\vec{V}_{0}(z)=U_{0}(z) \overrightarrow{e_{x}}$ ] and to a linear $z$ profile for the temperature. For $z$ between -1 and 1 , this basic state can be expressed as

$$
\begin{aligned}
& U_{0}(z)=1-z^{2}, \\
& T_{0}(z)=0.5(1-z) .
\end{aligned}
$$

If we now consider the spanwise magnetic field $\left(\overrightarrow{e_{B}}=\overrightarrow{e_{y}}\right)$, it can be shown that due to the current conservation in the $z$ direction, the potential induced current $-\vec{\nabla} \Phi=-\left(d \Phi_{0} / d z\right) \overrightarrow{e_{z}}$ will balance the directly induced current $\vec{V} \times \overrightarrow{e_{B}}=U_{0} \overrightarrow{e_{z}}$ so that the Lorentz force has no effect on the flow and the basic steady state is unchanged. (Note that for a vertical magnetic field, in contrast, there is a braking effect of the magnetic field on the flow. ${ }^{11}$ )

\section{B. Numerical approach}

We analyze the linear stability of the basic state (7) and (8) with respect to infinitesimal disturbances. The general solution of the problem is written as

$$
(\vec{V}, P, T, \Phi)=\left(\vec{V}_{0}, P_{0}, T_{0}, \Phi_{0}\right)+(\vec{v}, p, \theta, \phi),
$$

where $(\vec{v}, p, \theta, \phi)$ are the velocity, pressure, temperature, and electric potential perturbations, respectively. After substitution of Eq. (9) into the governing equations. (1)-(6) and linearization with respect to the small perturbations, we obtain

$$
\begin{aligned}
& \vec{\nabla} \cdot \vec{v}=0, \\
& \frac{\partial \vec{v}}{\partial t}+\left(\overrightarrow{V_{0}} \cdot \vec{\nabla}\right) \vec{v}+(\vec{v} \cdot \vec{\nabla}) \vec{V}_{0} \\
& \quad=-\vec{\nabla} p+\frac{1}{\operatorname{Re}} \nabla^{2} \vec{v}+\frac{\mathrm{Ra}}{8 \operatorname{Re}^{2} \operatorname{Pr}} \theta \overrightarrow{e_{z}}+\frac{\mathrm{Ha}^{2}}{\mathrm{Re}_{j}} \times \overrightarrow{e_{B}},
\end{aligned}
$$

$\vec{j}=-\vec{\nabla} \phi+\vec{v} \times \overrightarrow{e_{B}}$

$\frac{\partial \theta}{\partial t}+\overrightarrow{V_{0}} \cdot \vec{\nabla} \theta+\vec{v} \cdot \vec{\nabla} T_{0}=\frac{1}{\operatorname{RePr}} \nabla^{2} \theta$

$$
\nabla^{2} \phi=\overrightarrow{e_{B}} \cdot(\vec{\nabla} \times \vec{v})
$$

where $\vec{V}_{0}=\left(U_{0}, 0,0\right)$. In an extended layer, the disturbances can be expressed as normal modes in both $x$ and $y$ directions, 


$$
(\vec{v}, p, \theta, \phi)=(\vec{v}, p, \theta, \phi)(z) e^{i\left(k_{x} x+k_{y} y\right)+\omega t},
$$

where $k_{x}$ and $k_{y}$ are real wavenumbers in the longitudinal, $x$, and transverse, $y$, directions, respectively, and $\omega=\omega_{r}+i \omega_{i}$ is a complex eigenvalue. The real part $\omega_{r}$ represents an amplification rate and the imaginary part $\omega_{i}$ an oscillation frequency. The linearized equations (10)-(14) can be rewritten as

$$
\begin{aligned}
& i k_{x} u+i k_{y} v+D w=0 \\
& {\left[\frac{D^{2}-\left(k_{x}^{2}+k_{y}^{2}\right)}{\operatorname{Re}}-i k_{x} U_{0}(z)\right] u-D U_{0}(z) w-i k_{x} p} \\
& +\frac{\mathrm{Ha}^{2}}{\mathrm{Re}}(-u+D \phi)=\omega u, \\
& {\left[\frac{D^{2}-\left(k_{x}^{2}+k_{y}^{2}\right)}{\operatorname{Re}}-i k_{x} U_{0}(z)\right] v-i k_{y} p=\omega v,} \\
& {\left[\frac{D^{2}-\left(k_{x}^{2}+k_{y}^{2}\right)}{\operatorname{Re}}-i k_{x} U_{0}(z)\right] w-D p} \\
& +\frac{\mathrm{Ra}}{8 \operatorname{Re}^{2} \operatorname{Pr}} \theta+\frac{\mathrm{Ha}^{2}}{\operatorname{Re}}\left(-w-i k_{x} \phi\right)=\omega w, \\
& {\left[\frac{D^{2}-\left(k_{x}^{2}+k_{y}^{2}\right)}{\operatorname{RePr}}-i k_{x} U_{0}(z)\right] \theta-D T_{0}(z) w=\omega \theta,} \\
& {\left[D^{2}-\left(k_{x}^{2}+k_{y}^{2}\right)\right] \phi=u-i k_{x} w,}
\end{aligned}
$$

where $D=d / d z$. A generalized eigenvalue problem is obtained: $L X=\omega M X$, where $X=[\vec{v}(z), p(z), \theta(z), \phi(z)], L$ is a linear operator depending on $\mathrm{Pr}, \mathrm{Re}, \mathrm{Ra}, \mathrm{Ha}, k_{x}$, and $k_{y}$, and $M$ is a constant linear operator. This eigenvalue problem is discretized with the spectral Tau Chebyshev method and solved by the QZ algorithm of the NAG library. ${ }^{16}$ From the thresholds $\operatorname{Ra}_{0}\left(\operatorname{Pr}, \operatorname{Re}, \mathrm{Ha}, k_{x}, k_{y}\right)$ for which an eigenvalue has a real part equal to zero, whereas all the other eigenvalues have negative real parts, the critical Rayleigh number $\mathrm{Ra}_{c}$ is obtained by minimization with respect to $k_{x}$ and $k_{y}$ :

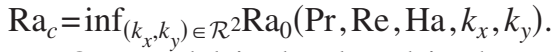

Our model is developed in the case of an infinite fluid domain. A question is raised about its validity for transversally confined situations. According to Burr and Müller, ${ }^{18}$ if the fluid is confined in the transverse direction by vertical walls perpendicular to the magnetic field, there will be damping effects. More precisely, the damping will depend on the electrical conductivity of these walls; Burr and Müller ${ }^{18}$ showed that for electrically insulated walls, the damping is less effective and decreases as the distance between the two walls increases, whereas for electrically well conducting walls, the damping is stronger and independent of the distance between the two walls. Our model is then strictly valid as the limit (for large widths) of transversally confined situations only for electrically insulating lateral walls.

\section{Energy balance}

Important information concerning the physical mechanisms involved in the development of the instabilities can be obtained from the calculation of the kinetic energy budget at critical parameter values. For that, the linearized NavierStokes equation (11) is multiplied by $\vec{v}^{*}$, the complex conjugate of $\vec{v}$, integrated along $z$, and then reduced to its real part. The resulting equation of energy budget expresses the rate of change of the fluctuating kinetic energy $K$ defined as $K=\int_{-1}^{1}\left(\vec{v} \cdot \vec{v}^{*} / 2\right) d z$

$$
\mathcal{R} e\left(\int_{-1}^{1} \frac{\partial \vec{v}}{\partial t} \cdot \vec{v}^{*} d z\right)=\frac{\partial K}{\partial t}=2 \omega_{r} K=K_{s}+K_{d}+K_{b}+K_{m},
$$

with

$$
\begin{aligned}
& K_{s}=-\mathcal{R} e\left(\int_{-1}^{1} w \frac{\partial U_{0}}{\partial z} u^{*} d z\right), \\
& K_{d}=\mathcal{R} e\left(\frac{1}{\operatorname{Re}} \int_{-1}^{1} \nabla^{2} \vec{v} \cdot \vec{v}^{*} d z\right), \\
& K_{b}=\mathcal{R} e\left(\frac{\operatorname{Ra}}{8 \operatorname{Re}^{2} \operatorname{Pr}} \int_{-1}^{1} \theta w^{*} d z\right), \\
& K_{m}=\mathcal{R} e\left[\frac{\operatorname{Ha}^{2}}{\operatorname{Re}} \int_{-1}^{1}\left(\vec{j} \times \overrightarrow{e_{B}}\right) \cdot \vec{v}^{*} d z\right] .
\end{aligned}
$$

$K_{s}$ represents the production of fluctuating kinetic energy by shear of the basic flow, $K_{d}$ represents the viscous dissipation of fluctuating kinetic energy, $K_{b}$ represents the production of fluctuating kinetic energy by buoyancy, and $K_{m}$ represents the dissipation of fluctuating kinetic energy by the magnetic forces. In these expressions, $\mathcal{R} e$ and the superscript $*$ denote the real part and the complex conjugate, respectively.

At threshold, the eigenvector used to calculate the energy budget is critical, i.e., associated with an eigenvalue of zero real part. This implies that $\partial K / \partial t$ is equal to zero. Moreover, since the eigenvectors are defined to within a multiplicative constant, this will also affect the different energy contributions. As a consequence, it is better to normalize the energy budget (22), and we use for that $-K_{d}=\left|K_{d}\right|$, which is always positive. A new equation involving the normalized energy terms $E_{s}, E_{b}$, and $E_{m}$ is thus obtained,

$$
E_{s}+E_{b}+E_{m}=1 \text {. }
$$

The magnetic term $E_{m}$ can also be split into two contributions, $E_{m, B}$ connected to the magnetic force $\left(\vec{v} \times \overrightarrow{e_{B}}\right) \times \overrightarrow{e_{B}}$ due to the directly induced current and $E_{m, \phi}$ connected to the magnetic force $-\vec{\nabla} \phi \times \overrightarrow{e_{B}}$ resulting from the electric potential. 


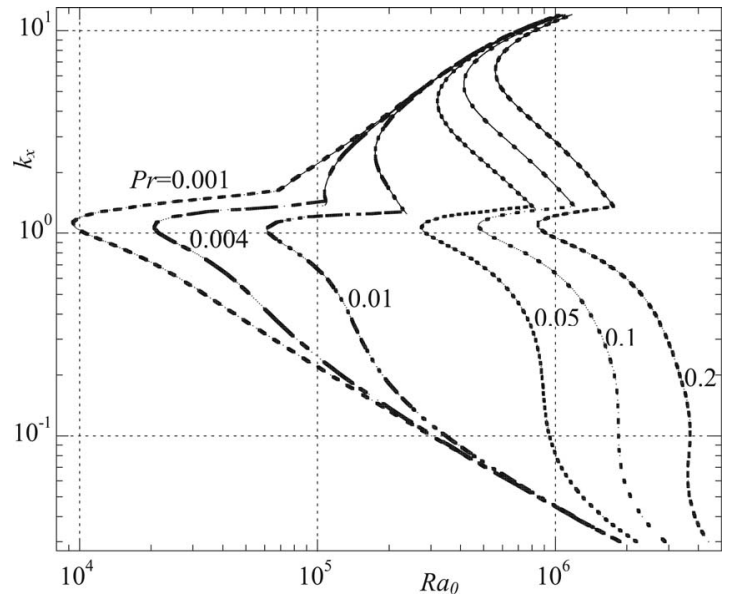

FIG. 1. Neutral stability curves $\left(\operatorname{Ra}_{0}\right.$ vs $\left.k_{x}\right)$ for the transverse $(T)$ modes in the PRB flow for different values of the Prandtl number Pr at $\mathrm{Re}=2000$. These curves have been found not to depend on the Hartmann number.

\section{LINEAR STABILITY RESULTS}

We first consider the neutral stability curves corresponding to the modes which are dominant in the PRB flow without magnetic field, i.e., the oscillatory transverse $(T)$ modes and the stationary longitudinal $(L)$ modes. The neutral curves giving $\mathrm{Ra}_{0}$ as a function of $k_{x}$ for the $(T)$ modes for different values of the Prandtl and Hartmann numbers and at $\operatorname{Re}=2000$ are plotted in Fig. 1. We see that the spanwise magnetic field has no effect on the neutral curves for the $(T)$ modes, as for each Prandtl number value these neutral curves do not change with an increase in Ha and remain identical to those obtained without magnetic field at $\mathrm{Ha}=0$. In contrast, the Prandtl number Pr has a clear effect on these neutral curves. When Pr is increased, the curves are moved to larger $\mathrm{Ra}$ values, and their aspect is modified with the creation of new local minimums. For small Pr values, only one minimum is observed for a wavenumber $k_{x}$ close to 1 ; for larger $\operatorname{Pr}$ values $(\operatorname{Pr} \geq 0.004)$, another local minimum appears at larger wavenumbers; and finally for $\operatorname{Pr}>0.1$ a third local minimum occurs at smaller wavenumbers. The existence of such multiple local minimums, which indicate the presence of different types of transverse unstable modes $(T)$, was already mentioned by Fujimura and Kelly ${ }^{19}$ in their study without magnetic field. These different transverse modes will be labeled as $\left(T_{1}\right),\left(T_{2}\right)$, and $\left(T_{3}\right)$, following their order of appearance when $\operatorname{Pr}$ is increased. Note that at $\mathrm{Re}=2000$ and in the range of Pr studied, the $\left(T_{3}\right)$ mode never becomes the dominant mode, whereas the $\left(T_{2}\right)$ mode becomes dominant when Pr exceeds a value that is between 0.05 and 0.1 .

The neutral curves giving $\mathrm{Ra}_{0}$ as a function of $k_{y}$ for the $(L)$ modes for different values of the Prandtl, Reynolds, and Hartmann numbers are plotted in Fig. 2. We see that these neutral curves are independent of the Prandtl and Reynolds numbers but very sensitive to the action of the spanwise magnetic field. When $\mathrm{Ha}$ is increased, the curves (with a single minimum which moves to smaller wavenumbers) are strongly moved to large values of Ra. This indicates a strong stabilization of these stationary longitudinal $(L)$ modes by the spanwise magnetic field, with an increase in the marginal

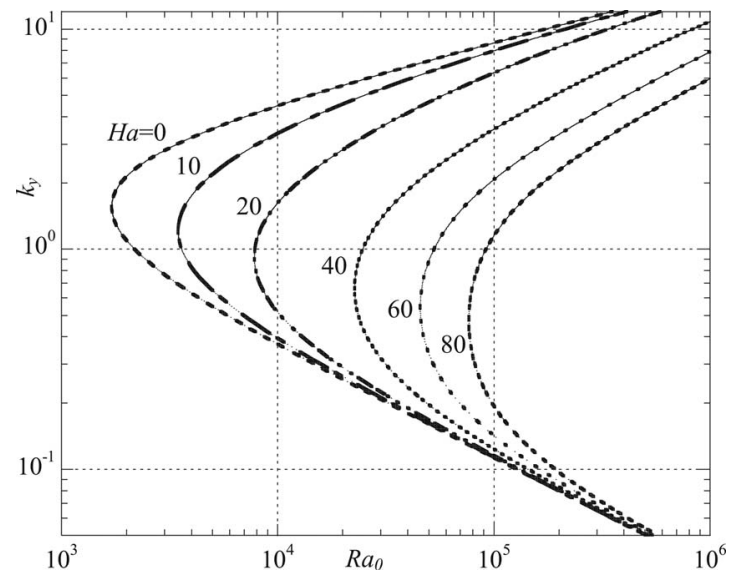

FIG. 2. Neutral stability curves $\left(\mathrm{Ra}_{0}\right.$ vs $\left.k_{y}\right)$ for the longitudinal $(L)$ modes in the PRB flow for different values of the Hartmann number Ha. These curves have been found not to depend on the Prandtl and Reynolds numbers.

cell size. Scaling laws can be derived for these variations in $\mathrm{Ra}_{c}$ and $k_{y, c}$ in the large Ha range. It is thus found that $\mathrm{Ra}_{c} \sim \mathrm{Ha}^{2}$ and $k_{y, c} \sim \mathrm{Ha}^{-0.5}$. The scaling law in $\mathrm{Ha}^{2}$ for $\mathrm{Ra}_{c}$ is similar to that found by Chandrasekhar ${ }^{20}$ in the study of the pure Rayleigh-Bénard situation in the presence of a vertical magnetic field. The scaling laws for the critical wavenumbers, however, are different: a decrease is obtained in our case with a spanwise magnetic field, whereas an increase $\left(k_{y, c} \sim \mathrm{Ha}^{1 / 3}\right)$ is obtained by Chandrasekhar for a vertical magnetic field.

The selective effect of the spanwise magnetic field on the transverse $(T)$ modes and longitudinal $(L)$ modes can also be stated by simple analytical derivations. If we take the curl of the linearized Navier-Stokes equation (11), the magnetic force term is changed to

$$
\frac{\mathrm{Ha}^{2}}{\operatorname{Re}} \vec{\nabla} \times\left(\vec{j} \times \overrightarrow{e_{B}}\right)
$$

Taking into account current conservation and the fact that the magnetic field is constant, we can write

$$
\begin{aligned}
\vec{\nabla} \times\left(\vec{j} \times \overrightarrow{e_{B}}\right) & =\vec{j}\left(\vec{\nabla} \cdot \overrightarrow{e_{B}}\right)-(\vec{j} \cdot \vec{\nabla}) \overrightarrow{e_{B}}-\overrightarrow{e_{B}}(\vec{\nabla} \cdot \vec{j})+\left(\overrightarrow{e_{B}} \cdot \vec{\nabla}\right) \vec{j} \\
& =\left(\overrightarrow{e_{B}} \cdot \vec{\nabla}\right) \vec{j} .
\end{aligned}
$$

With $\vec{\nabla}=i k_{x} \overrightarrow{e_{x}}+i k_{y} \overrightarrow{e_{y}}+\overrightarrow{e_{z}} D$ and $\overrightarrow{e_{B}}=\overrightarrow{e_{y}}$ (spanwise magnetic field), we finally obtain

$$
\vec{\nabla} \times\left(\vec{j} \times \overrightarrow{e_{B}}\right)=i k_{y} \vec{j}
$$

Applying Eq. (30) to the $(T)$ modes $\left(k_{x} \neq 0\right.$ and $\left.k_{y}=0\right)$, we obtain

$$
\vec{\nabla} \times\left(\vec{j} \times \overrightarrow{e_{B}}\right)=\overrightarrow{0}
$$

so that $\vec{j} \times \overrightarrow{e_{B}}$, which can be expressed as a gradient and introduced in a modified pressure, has no effect on these modes. In contrast, for the $(L)$ modes $\left(k_{x}=0\right.$ and $\left.k_{y} \neq 0\right)$, we obtain 


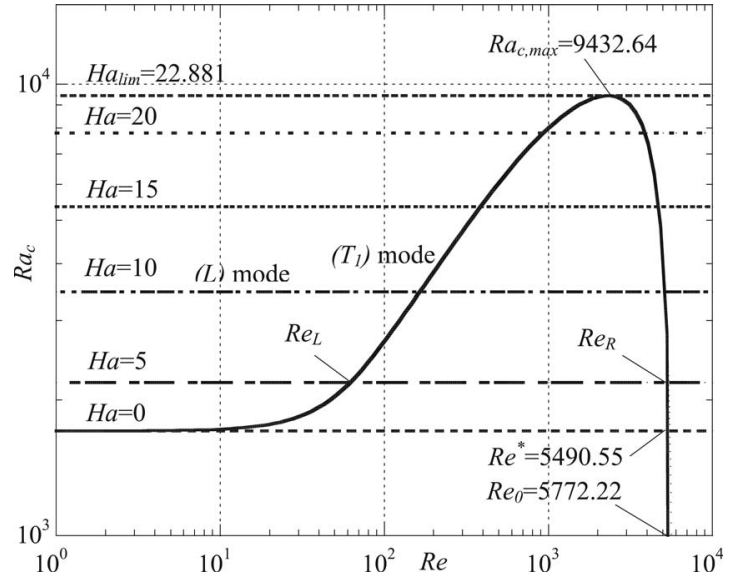

FIG. 3. Variation in the critical Rayleigh number $\mathrm{Ra}_{c}$ as a function of $\mathrm{Re}$ for the transverse $\left(T_{1}\right)$ modes and the longitudinal $(L)$ modes in the PRB flow for $\operatorname{Pr}=0.001$ and different values of the Hartmann number Ha. Only the thresholds for the $(L)$ modes depend on Ha.

$$
\vec{\nabla} \times\left(\vec{j} \times \overrightarrow{e_{B}}\right) \neq \overrightarrow{0}
$$

indicating that the magnetic force is active in this case. This approach confirms that the spanwise magnetic field has a stabilizing effect on the longitudinal $(L)$ modes but no effect on the transverse $(T)$ modes.

In the following, we will see how this selective effect of the spanwise magnetic field will affect the dominant critical modes. Three values of the Prandtl number will be successively chosen.

\section{A. Critical curves for $\mathrm{Pr}=0.001$}

The critical curves for $\operatorname{Pr}=0.001$ are given in Fig. 3 through the plots of the critical Rayleigh number $\mathrm{Ra}_{c}$ as a function of the Reynolds number Re for different values of the Hartmann number Ha. Our investigations for $\mathrm{Pr}=0.001$ have shown that the neutral stability curves for the $(T)$ modes present a single minimum in the range of Re studied. Only one type of transverse instabilities, namely, the $\left(T_{1}\right)$ modes, will affect the fluid layer. As shown in Sec. III, the transverse modes are not affected by the magnetic field. The critical values of $\mathrm{Ra}$ for the $\left(T_{1}\right)$ instability then remain independent of $\mathrm{Ha}$ but will depend on Re. As shown in Fig. 3, $\mathrm{Ra}_{c}$ increases with $\mathrm{Re}$, reaches a maximum of $\mathrm{Ra}_{c, \text { max }}=9432.64$ at $\mathrm{Re}_{\max }=2320.27$, and then decreases when $\mathrm{Re}$ is further increased, until $\mathrm{Ra}_{c}=0$ which is reached for $\operatorname{Re}_{0}=5772.22$. This value $\operatorname{Re}_{0}$ is the critical Reynolds number for an isothermal plane Poiseuille flow (onset of Tollmien-Schlichting waves).

Concerning the $(L)$ modes, they are not affected by the Poiseuille flow, and the critical curves are then horizontal lines in the $(\mathrm{Re}, \mathrm{Ra})$ plane. They are, however, stabilized by the action of the spanwise magnetic field so that these horizontal lines will move up when Ha will be increased (Fig. 3).

We can now compare the critical thresholds of the $\left(T_{1}\right)$ and $(L)$ modes in order to see how the dominant modes change when $\mathrm{Ha}$ is increased. Note first that without magnetic field (the case $\mathrm{Ha}=0$ ), the critical curves corresponding to the $\left(T_{1}\right)$ and $(L)$ modes in the $(\mathrm{Re}, \mathrm{Ra})$ plane intersect at a
TABLE I. Variation with Ha of the domain where the $(L)$ modes are dominant for $\operatorname{Pr}=0.001$. For each value of $\mathrm{Ha}$ are given the $\mathrm{Re}$ extent of this domain (left border $\mathrm{Re}_{L}$, right border $\mathrm{Re}_{R}$, and extent $\Delta \mathrm{Re}$ ) and the critical Rayleigh number $\mathrm{Ra}_{c}$.

\begin{tabular}{ccccc}
\hline \hline $\mathrm{Ha}$ & $\mathrm{Ra}_{c}$ & $\mathrm{Re}_{L}$ & $\mathrm{Re}_{R}$ & $\Delta \mathrm{Re}$ \\
\hline 1 & 1727.66 & 11.706 & 5486.81 & 5475.10 \\
2 & 1786.91 & 23.551 & 5476.33 & 5452.78 \\
5 & 2185.22 & 61.982 & 5404.42 & 5342.44 \\
8 & 2870.39 & 113.593 & 5274.52 & 5160.93 \\
10 & 3462.19 & 164.243 & 5155.12 & 4990.88 \\
12 & 4151.04 & 233.674 & 5006.22 & 4772.55 \\
15 & 5354.59 & 387.240 & 4713.33 & 4326.09 \\
18 & 6753.37 & 643.082 & 4295.14 & 3652.05 \\
20 & 7791.43 & 929.888 & 3888.66 & 2958.77 \\
21 & 8341.70 & 1147.24 & 3609.05 & 2461.81 \\
22 & 8912.68 & 1477.32 & 3216.36 & 1739.04 \\
22.881 & 9432.64 & 2320.27 & 2320.27 & 0 \\
\hline \hline
\end{tabular}

single crossing point denoted by $\left(\mathrm{Re}^{*}, \mathrm{Ra}^{*}\right)$. For $\operatorname{Pr}=0.001$, we have found that this point corresponds to $\mathrm{Re}^{*}=5490.55$ and $\mathrm{Ra}^{*}=1707.76$, as already mentioned by Fujimura and Kelly ${ }^{19}$ and Fakhfakh et al. ${ }^{11}$ In contrast, in the presence of a spanwise magnetic field (at the condition that the Hartmann number remains smaller than a limit value $\left.\mathrm{Ha}_{\text {lim }}\right)$, the $\left(T_{1}\right)$ and $(L)$ critical curves will intersect at two different points. These points are denoted by $\left(\mathrm{Re}_{L}, \mathrm{Ra}_{L}\right)$ and $\left(\mathrm{Re}_{R}, \mathrm{Ra}_{R}\right)$ with $\operatorname{Re}_{L}<\operatorname{Re}_{R}$. According to Fig. 3, it is clear that $\operatorname{Re}_{L}<\operatorname{Re}_{\text {max }}$ $<\operatorname{Re}_{R}$ and that $\mathrm{Ra}_{L}=\mathrm{Ra}_{R}=\mathrm{Ra}_{c}(\mathrm{Ha})$, where $\mathrm{Ra}_{c}(\mathrm{Ha})$ is the critical Rayleigh number for the $(L)$ modes, which only depends on Ha.

Figure 3 clearly shows the domains (Re ranges) where each mode is dominant. Without magnetic field, the $(L)$ modes are dominant in a large range of Re values, $0 \leq \mathrm{Re}$ $\leq \mathrm{Re}^{*}=5490.55$, whereas the $\left(T_{1}\right)$ modes are dominant only very locally, close to $\operatorname{Re}_{0}, \quad \mathrm{Re}^{*}=5490.55 \leq \mathrm{Re} \leq \mathrm{Re}_{0}$ $=5772.22$. Because of the selective effect of the spanwise magnetic field, which only stabilizes the $(L)$ modes, the Re range where these $(L)$ modes are dominant will progressively shrink as Ha is increased, which will automatically induce an increase in the domain where the $\left(T_{1}\right)$ modes are dominant. As shown in Fig. 3, for a given value of $\mathrm{Ha}<\mathrm{Ha}_{\text {lim }}$, the $(L)$ modes are now dominant only in the range of $\operatorname{Re}_{L}<\mathrm{Re}$ $<\mathrm{Re}_{R}$, whereas the $\left(T_{1}\right)$ modes are dominant for $0<\mathrm{Re}$ $<\operatorname{Re}_{L}$ and $\operatorname{Re}_{R}<\mathrm{Re} \leq \mathrm{Re}_{0}$. Moreover, when Ha is increased up to $\mathrm{Ha}_{\text {lim }}$, the increase in $\mathrm{Ra}_{c}$ will induce both the increase in $\mathrm{Re}_{L}$ and the decrease in $\mathrm{Re}_{R}$. For example, for $\mathrm{Ha}=5$, $\mathrm{Ra}_{c}=2185.22, \mathrm{Re}_{L}=61.982$, and $\mathrm{Re}_{R}=5404.42$, and for $\mathrm{Ha}=10, \mathrm{Ra}_{c}=3462.19, \mathrm{Re}_{L}=164.243$, and $\operatorname{Re}_{R}=5155.12$ (see Table I). The extent of the domain where the $(L)$ modes are dominant, which is measured by $\Delta \mathrm{Re}=\mathrm{Re}_{R}-\mathrm{Re}_{L}$, will then decrease from 5342.44 to 4990.88 when $\mathrm{Ha}$ is increased from five to ten. The decrease in $\Delta \mathrm{Re}$ with the increase in $\mathrm{Ha}$ is shown in details in Table I. When Ha tends toward $\mathrm{Ha}_{\text {lim }}=22.881$, the crossing points $\left(\mathrm{Re}_{L}, \mathrm{Ra}_{L}\right)$ and $\left(\mathrm{Re}_{R}, \mathrm{Ra}_{R}\right)$ get closer until they merge at $\mathrm{Ha}_{\text {lim }}$, which corresponds to $\Delta \mathrm{Re}=0$. Beyond $\mathrm{Ha}_{\text {lim }}$, the $(L)$ modes are no more dominant, 


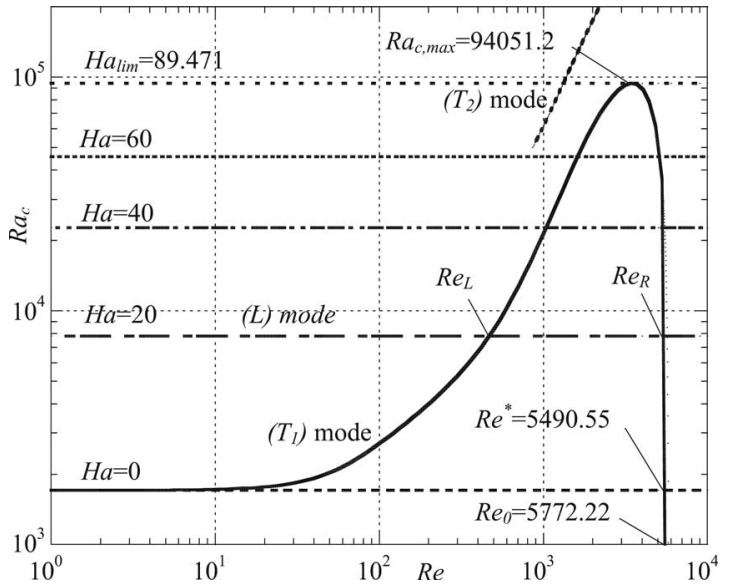

FIG. 4. Variation in the critical Rayleigh number $\mathrm{Ra}_{c}$ as a function of Re for the transverse $\left(T_{1}\right)$ and $\left(T_{2}\right)$ modes and the longitudinal $(L)$ modes in the PRB flow for $\operatorname{Pr}=0.01$ and different values of the Hartmann number Ha. Only the thresholds for the $(L)$ modes depend on Ha.

and only the $\left(T_{1}\right)$ modes will be responsible for the destabilization of the PRB flow from $\operatorname{Re}=0$ to $\operatorname{Re}=\operatorname{Re}_{0}=5772.22$.

The results obtained for $\operatorname{Pr}=0.001$ show that by applying a spanwise magnetic field and adjusting the value of $\mathrm{Ha}$, it is possible to select the type of instability which will be first triggered in the PRB flow. In particular, a possibility is given to get rid of the $(L)$ modes, and in applications where Re remains small, this can be obtained for relatively small magnetic field intensities.

\section{B. Critical curves for $\mathrm{Pr}=0.01$}

The value $\operatorname{Pr}=0.01$ corresponds to liquid sodium used as a coolant in fast breeder fission reactors. ${ }^{21,22}$ We can first note that for this value of $\mathrm{Pr}$, as shown in Fig. 1, the neutral stability curves for the transverse modes have two local minimums instead of a single one for $\operatorname{Pr}=0.001$. The $\left(T_{2}\right)$ mode corresponding to the second minimum, however, never becomes the most critical mode for the transverse instabilities. Such a feature is illustrated in Fig. 4 where the critical curves for $\operatorname{Pr}=0.01$ are plotted for the longitudinal $(L)$ modes as well as for both $\left(T_{1}\right)$ and $\left(T_{2}\right)$ transverse modes. As a consequence, the $\left(T_{2}\right)$ modes will not affect the competition between the transverse and longitudinal modes, which will be similar to what was observed for $\operatorname{Pr}=0.001$. For $\mathrm{Ha}<\mathrm{Ha}_{\text {lim }}$, the $\left(T_{1}\right)$ modes will be dominant in the Re ranges, $0<\operatorname{Re}<\operatorname{Re}_{L}$ and $\operatorname{Re}_{R}<\operatorname{Re} \leq \operatorname{Re}_{0}$, while the $(L)$ modes will prevail for $\operatorname{Re}_{L}<\operatorname{Re}<\operatorname{Re}_{R}$ (Fig. 4). Moreover, for $\mathrm{Ha} \geq \mathrm{Ha}_{\text {lim }}$, only the $\left(T_{1}\right)$ modes are involved in the destabilization of the PRB flow. The characteristic values $\mathrm{Re}_{L}$ and $\mathrm{Re}_{R}$ (functions of $\mathrm{Ha}$ ) as well as $\mathrm{Ha}_{\text {lim }}$, however, have changed compared to the case at $\operatorname{Pr}=0.001$. This is due to the stronger increase with $\mathrm{Re}$ of the critical curve for the $\left(T_{1}\right)$ modes, with a peak value at $\mathrm{Ra}_{c, \text { max }}=94051.2$ compared to $\mathrm{Ra}_{c, \max }=9432.64$ for $\operatorname{Pr}=0.001$. Stronger values of Ha are then necessary to decrease the Re range where the $(L)$ modes are dominant (Table II), and the value of $\mathrm{Ha}_{\text {lim }}$ has increased to 89.471 compared to 22.881 for $\operatorname{Pr}=0.001$.
TABLE II. Variation with Ha of the domain where the $(L)$ modes are dominant for $\operatorname{Pr}=0.01$. For each value of $\mathrm{Ha}$ are given the $\mathrm{Re}$ extent of this domain (left border $\mathrm{Re}_{L}$, right border $\mathrm{Re}_{R}$, and extent $\Delta \mathrm{Re}$ ) and the critical Rayleigh number $\mathrm{Ra}_{c}$.

\begin{tabular}{ccccc}
\hline \hline $\mathrm{Ha}$ & $\mathrm{Ra}_{c}$ & $\mathrm{Re}_{L}$ & $\mathrm{Re}_{R}$ & $\Delta \mathrm{Re}$ \\
\hline 1 & 1727.66 & 11.795 & 5751.67 & 5739.88 \\
10 & 3462.19 & 157.833 & 5731.08 & 5573.25 \\
20 & 7791.43 & 467.889 & 5678.75 & 5210.86 \\
30 & 14218.6 & 764.384 & 5598.37 & 4833.98 \\
40 & 22676.8 & 1040.27 & 5487.10 & 4446.82 \\
50 & 33149.7 & 1318.58 & 5339.04 & 4020.46 \\
60 & 45630.2 & 1618.46 & 5143.57 & 3525.11 \\
70 & 60113.6 & 1965.93 & 4879.39 & 2913.46 \\
80 & 76597.5 & 2420.34 & 4490.51 & 2070.17 \\
85 & 85588.8 & 2750.57 & 4187.30 & 1436.73 \\
89.471 & 94051.2 & 3479.40 & 3479.40 & 0 \\
\hline \hline
\end{tabular}

\section{Critical curves for $\mathrm{Pr}=0.1$}

For $\operatorname{Pr}=0.1$, the neutral stability curves for the transverse modes still have two local minimums [corresponding to the $\left(T_{1}\right)$ and $\left(T_{2}\right)$ modes $]$ as for $\operatorname{Pr}=0.01$. This time, however, the $\left(T_{2}\right)$ mode may become the dominant transverse mode, as shown in Fig. 1 for $\operatorname{Re}=2000$. Our calculations for $\operatorname{Pr}=0.1$ have shown that this is the case for Re values in the range of $\operatorname{Re}_{l}=320.612<\operatorname{Re}<\operatorname{Re}_{h}=2606.40$, which correspond to $\operatorname{Ra}_{c}$ varying between $\mathrm{Ra}_{l}=35388.0$ and $\mathrm{Ra}_{h}=590020$. This is depicted in Fig. 5 where the critical curves for the $(L),\left(T_{1}\right)$, and $\left(T_{2}\right)$ modes are plotted for $\operatorname{Pr}=0.1$.

As in the previous cases, the stabilization of the $(L)$ mode by the spanwise magnetic field will change the order of appearance of the different modes as $\mathrm{Ha}$ is increased. For $\operatorname{Pr}=0.1$, however, the situation is still different because of the two transverse modes involved and the very strong increase with $\mathrm{Re}$ of the thresholds for these transverse modes [the peak value for the $\left(T_{1}\right)$ mode is now at $\mathrm{Ra}_{c}=650$ 065]. Four intervals of $\mathrm{Ha}$ can be distinguished (Fig. 5). Two of the limit values are $\mathrm{Ha}_{l}$ and $\mathrm{Ha}_{h}$, which are defined by $\mathrm{Ra}_{c}\left(\mathrm{Ha}_{l}\right)=\mathrm{Ra}_{l}$

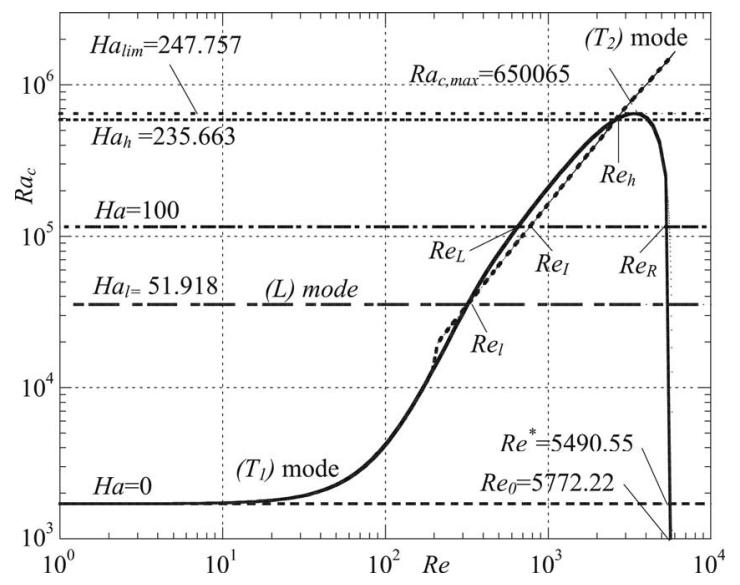

FIG. 5. Variation in the critical Rayleigh number $\mathrm{Ra}_{c}$ as a function of Re for the transverse $\left(T_{1}\right)$ and $\left(T_{2}\right)$ modes and the longitudinal $(L)$ modes in the $\mathrm{PRB}$ flow for $\mathrm{Pr}=0.1$ and different values of the Hartmann number Ha. Only the thresholds for the $(L)$ modes depend on Ha. 
TABLE III. Variation with Ha of the domain where the $(L)$ modes are dominant for $\operatorname{Pr}=0.1$. For each value of $\mathrm{Ha}$ are given the $\mathrm{Re}$ extent of this domain (left border $\mathrm{Re}_{L}$, right border $\mathrm{Re}_{R}$, and extent $\Delta \mathrm{Re}$ ) and the critical Rayleigh number $\mathrm{Ra}_{c}$.

\begin{tabular}{lcccc}
\hline \hline Ha & $\mathrm{Ra}_{c}$ & $\mathrm{Re}_{L}$ & $\mathrm{Re}_{R}$ & $\Delta \mathrm{Re}$ \\
\hline 20 & 7791.43 & 149.333 & 5758.48 & 5609.15 \\
40 & 22676.9 & 257.165 & 5732.42 & 5475.26 \\
51.918 & 35388.0 & 320.612 & 5709.94 & 5389.33 \\
60 & 45630.5 & $386.294^{\mathrm{a}}$ & 5691.60 & 5305.31 \\
100 & 115559 & $771.243^{\mathrm{a}}$ & 5561.76 & 4790.52 \\
150 & 247842 & $1365.67^{\mathrm{a}}$ & 5288.34 & 3922.66 \\
200 & 429857 & $2062.87^{\mathrm{a}}$ & 4815.69 & 2752.82 \\
230 & 562900 & $2517.44^{\mathrm{a}}$ & 4305.45 & 1788.01 \\
235.663 & 590020 & 2606.40 & 4155.69 & 1549.29 \\
240 & 611218 & 2767.27 & 4011.46 & 1244.19 \\
245 & 636121 & 3026.85 & 3770.94 & 744.088 \\
247.757 & 650065 & 3400.30 & 3400.30 & 0 \\
\hline \hline
\end{tabular}

${ }^{\mathrm{a}} \mathrm{Re}_{I}$ (which is the true left border in this case) is given in place of $\operatorname{Re}_{L}$.

and $\mathrm{Ra}_{c}\left(\mathrm{Ha}_{h}\right)=\mathrm{Ra}_{h}$, respectively. For $\mathrm{Ha} \leq \mathrm{Ha}_{l}=51.918$ the most unstable modes are the $\left(T_{1}\right)$ modes or the $(L)$ modes, and the domains of Re where they are dominant are defined as previously from the values of $\mathrm{Re}_{L}$ and $\mathrm{Re}_{R}$. For $\mathrm{Ha}_{l}<\mathrm{Ha}<\mathrm{Ha}_{h}=235.663$, the $\left(T_{2}\right)$ mode will also be involved. In this case, we have to define the crossing point $\left(\mathrm{Re}_{I}, \mathrm{Ra}_{I}\right)$ between the stability curves for the $\left(T_{2}\right)$ and $(L)$ modes, which is such that $\operatorname{Re}_{L}<\mathrm{Re}_{I}<\mathrm{Re}_{R}$ (see the case $\mathrm{Ha}=100$ in Fig. 5). As a consequence, the $(L)$ modes become the dominant mode only for $\operatorname{Re}_{I}<\operatorname{Re}<\operatorname{Re}_{R}$ (Table III), whereas the transverse modes prevail outside this Re range, the $\left(T_{1}\right)$ modes for $0<\operatorname{Re}<\operatorname{Re}_{l}$ and $\operatorname{Re}_{R}<\operatorname{Re} \leq \operatorname{Re}_{0}$ and the $\left(T_{2}\right)$ modes for $\operatorname{Re}_{l}<\operatorname{Re}<\operatorname{Re}_{I}$. Note that the extent of the domain where the $\left(T_{2}\right)$ modes prevail, i.e., $\mathrm{Re}_{I}-\mathrm{Re}_{l}$, increases with $\mathrm{Ha}$ due to the increase in $\mathrm{Re}_{I}$. For $\mathrm{Ha}_{h}<\mathrm{Ha}$ $<\mathrm{Ha}_{\text {lim }}=247.757$, the situation is still a little changed because the extent of the domain where the $\left(T_{2}\right)$ modes are dominant becomes maximum (equal to $\mathrm{Re}_{h}-\mathrm{Re}_{l}=2285.85$ ) and independent of Ha. Furthermore, the domain where the (L) modes are the most critical still shrinks and corresponds to $\operatorname{Re}_{L}<\operatorname{Re}<\operatorname{Re}_{R}$, whereas the domain corresponding to the $\left(T_{1}\right)$ modes is further increased by the addition of a new narrow $\mathrm{Re}$ range, $\mathrm{Re}_{h}<\mathrm{Re}<\mathrm{Re}_{L}$. Finally when $\mathrm{Ha} \geq \mathrm{Ha}_{\text {lim }}$, the PRB flow is only affected by the transverse modes: the $\left(T_{1}\right)$ modes for $0<\operatorname{Re}<\operatorname{Re}_{l}$ and $\operatorname{Re}_{h}<\operatorname{Re} \leq \operatorname{Re}_{0}$ and the $\left(T_{2}\right)$ modes for $\operatorname{Re}_{l}<\operatorname{Re}<\operatorname{Re}_{h}$. Note that the variation with $\mathrm{Ha}$ of the Re domain where the $(L)$ modes prevail is also shown in Table III.

For $\operatorname{Pr}=0.1$, the spanwise magnetic field has still been found to favor the onset of transverse instabilities in the PRB flow. As shown in the introduction, this is an important point for practical applications as CVD and electronic device cooling. It has also been shown that the transverse $\left(T_{2}\right)$ modes, which have higher critical wavenumbers and wave speeds than the $\left(T_{1}\right)$ modes, are the preferred modes in some parameter range. Further numerical simulations performed for larger Prandtl numbers $(\operatorname{Pr} \geq 0.2)$ have shown that the dominant unstable modes are globally similar to those corre-

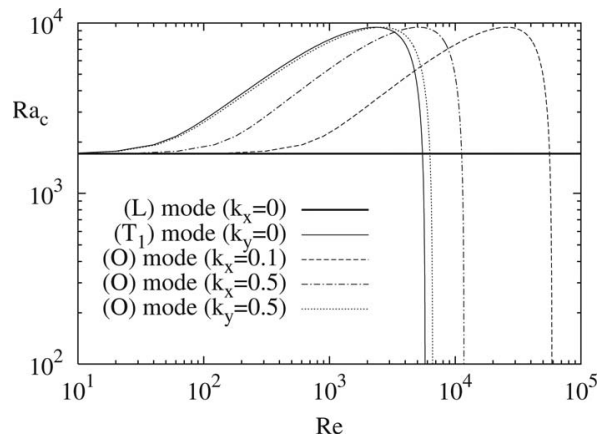

FIG. 6. Critical Rayleigh number $\mathrm{Ra}_{c}$ as a function of the Reynolds number $\mathrm{Re}$ for the PRB flow without magnetic field $(\mathrm{Ha}=0)$ : longitudinal $(L)$ mode $\left(k_{x}=0\right)$, transverse $\left(T_{1}\right)$ mode $\left(k_{y}=0\right)$, and oblique $(O)$ modes obtained by the Squire transformation for fixed values of $k_{x}\left(k_{x}=0.1\right.$ and 0.5$)$ or $k_{y}$ $\left(k_{y}=0.5\right)$. The results are obtained for $\operatorname{Pr}=0.001$.

sponding to the case $\operatorname{Pr}=0.1$. As illustrated in Fig. 1 for $\operatorname{Pr}=0.2$, the neutral stability curves for the transverse modes have now three local minimums corresponding to the three transverse modes $\left(T_{1}\right),\left(T_{2}\right)$, and $\left(T_{3}\right)$ which can develop in a PRB flow. The $\left(T_{3}\right)$ mode, however, never becomes the dominant mode for moderate $\operatorname{Pr}$ so that the competition between the modes will only concern the longitudinal $(L)$ mode and the transverse $\left(T_{1}\right)$ and $\left(T_{2}\right)$ modes, as for $\operatorname{Pr}=0.1$. Note that the main modification will be the extension of the domain where the $\left(T_{2}\right)$ modes are dominant.

\section{Fully three-dimensional disturbances}

The previous results have clearly shown that the spanwise magnetic field has a selective stabilizing effect by delaying the appearance of the longitudinal unstable $(L)$ modes, whereas the thresholds for the transverse $(T)$ modes remain unchanged in comparison with the case of $\mathrm{Ha}=0$. A question remains about the stabilization of the oblique $(O)$ modes which correspond to fully three-dimensional disturbances $\left(k_{x} \neq 0\right.$ and $\left.k_{y} \neq 0\right)$.

In the usual PRB flow (without magnetic field), as shown by Gage and Reid ${ }^{23}$ and later used by Jung et al. ${ }^{24}$ and $\mathrm{Hu}$ et al., ${ }^{25}$ there is a Squire transformation which allows one to deduce the critical curves for the oblique $(O)$ modes from the curves obtained for the transverse $(T)$ modes. This Squire transformation can be expressed as $\mathrm{Ra}_{O}=\mathrm{Ra}, k_{x} \mathrm{Re}_{O}=k \mathrm{Re}$, and $k^{2}=k_{x}^{2}+k_{y}^{2}$, where $\mathrm{Ra}$ and $\mathrm{Re}$ are associated with the transverse modes (with a wave vector $k$ along $x$ ) and $\mathrm{Ra}_{O}$ and $\operatorname{Re}_{O}$ are associated with the oblique modes (for arbitrary orientations of the wave vector determining arbitrary values of $k_{x}$ and $k_{y}$ ). The critical curves obtained by the Squire transformation for different oblique modes $\left(k_{x}=0.1, k_{x}=0.5\right.$, and $\left.k_{y}=0.5\right)$ are plotted in Fig. 6 together with the critical curves for the $(L)$ and $\left(T_{1}\right)$ modes. (These curves have been verified to exactly fit those obtained numerically.) We clearly see that without magnetic field, the oblique modes are never the dominant modes. It is difficult, however, to guess whether these modes will become dominant when a spanwise magnetic field is applied. Moreover, there is no Squire transformation valid in the case with magnetic field.

Numerical calculations have then been performed to de- 


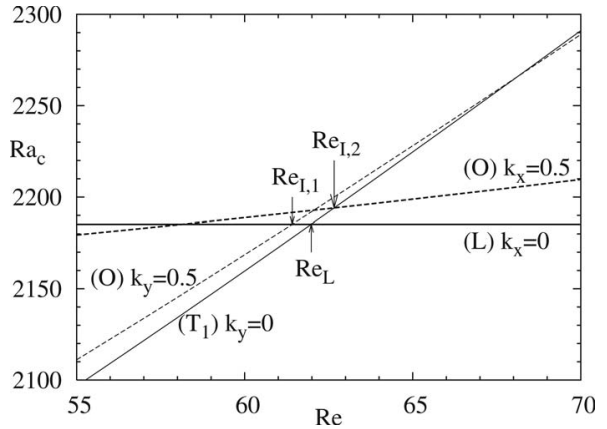

FIG. 7. Critical Rayleigh number $\mathrm{Ra}_{c}$ as a function of the Reynolds number $\mathrm{Re}$ for the PRB flow for $\mathrm{Ha}=5$ : longitudinal $(L)$ mode $\left(k_{x}=0\right)$, transverse $\left(T_{1}\right)$ mode $\left(k_{y}=0\right)$, and oblique $(O)$ modes obtained for $k_{x}=0.5$ and for $k_{y}=0.5$. This figure illustrates the definition of the Reynolds numbers $\operatorname{Re}_{I, 1}$ [intersection between the $(0)$ and $(L)$ modes] and $\mathrm{Re}_{I, 2}$ [intersection between the $(O)$ and $(T)$ modes] and shows that $\operatorname{Re}_{I, 1}<\operatorname{Re}_{L}<\operatorname{Re}_{I, 2}$ in this case, indicating that the oblique modes are not dominant modes. The results are obtained for $\operatorname{Pr}=0.001$.

termine the critical thresholds for the oblique $(O)$ modes. For that, we solve the generalized eigenvalue system (16)-(21) for $k_{x}$ fixed with a minimization with respect to $k_{y}$ or for $k_{y}$ fixed with a minimization with respect to $k_{x}$. We first present some results obtained for $\mathrm{Pr}=0.001$ and $\mathrm{Ha}=5$ in Fig. 7. In this figure, the critical curves for the $(L)$ and $\left(T_{1}\right)$ modes are plotted together with those for the oblique modes at $k_{x}=0.5$ and at $k_{y}=0.5$ in the neighborhood of the crossing point between the $\left(T_{1}\right)$ and $(L)$ modes $\left(\operatorname{Re}_{L}=61.982\right)$. We clearly see that the critical curves for the oblique modes intersect the critical curve for the $\left(T_{1}\right)$ mode before that for the $(L)$ mode. This indicates that in this particular case, the two chosen oblique modes are not the dominant modes. In order to check this in a more general way, it is interesting to define the intersection points between the curves for the $(O)$ and $(L)$ modes (denoted as $\operatorname{Re}_{I, 1}$ ) and between the curves for the $(O)$ and $(T)$ modes (denoted as $\operatorname{Re}_{I, 2}$ ). As is clear from Fig. 7 , the oblique modes will not be the dominant modes if $\operatorname{Re}_{I, 1}<\operatorname{Re}_{L}<\operatorname{Re}_{I, 2}$, i.e., $\operatorname{Re}_{I, 1}-\operatorname{Re}_{L}<0$ and $\operatorname{Re}_{I, 2}-\operatorname{Re}_{L}>0$. In fact, it is enough to verify one of these inequalities, and we have chosen to check the first one for oblique modes with fixed $k_{y}$ and the second one for oblique modes with fixed $k_{x}$.

The positions of the $\mathrm{Re}_{L}, \mathrm{Re}_{I, 1}$, and $\mathrm{Re}_{I, 2}$ points have been determined through numerical calculations for a large set of oblique modes $\left(0<k_{x} \leq 1\right.$ and $\left.0<k_{y} \leq 1\right)$ and for different Hartmann and Prandtl numbers. The results for $\operatorname{Pr}=0.001$, expressed either as $\operatorname{Re}_{I, 1}-\operatorname{Re}_{L}$ as a function of $k_{y}$ or as $\operatorname{Re}_{I, 2}-\operatorname{Re}_{L}$ as a function of $k_{x}$, are given in Fig. 8 for three values of the Hartmann number $(\mathrm{Ha}=0.5,1$, and 5). We clearly see that for all these cases, the values of $\mathrm{Re}_{I, 1}-\mathrm{Re}_{L}$ remain negative, whereas those of $\operatorname{Re}_{I, 2}-\mathrm{Re}_{L}$ are positive. Moreover, the values of $\left|\operatorname{Re}_{I, 1}-\operatorname{Re}_{L}\right|$ and $\mathrm{Re}_{I, 2}-\mathrm{Re}_{L}$ are the smallest for slightly oblique modes (close to transverse or close to longitudinal) and for small Hartmann numbers, and they increase with $k_{x}, k_{y}$, and Ha. Similar curves have also been obtained for $\operatorname{Pr}=0.01$ and $\operatorname{Pr}=0.1$; the main difference is that the increase with $k_{x}, k_{y}$, and $\mathrm{Ha}$ is smaller when $\mathrm{Pr}$ is increased. According to these results, it can be stated that the oblique $(O)$ modes corresponding to fully three-dimensional disturbances never become the dominant modes. As a conse-

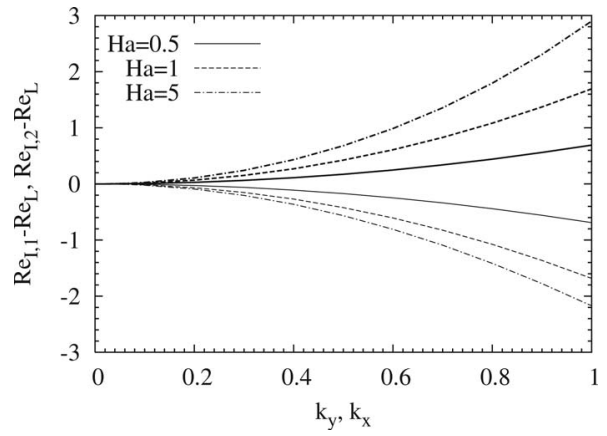

FIG. 8. Reynolds number differences $\mathrm{Re}_{I, 1}-\mathrm{Re}_{L}$ and $\mathrm{Re}_{I, 2}-\mathrm{Re}_{L}$ (see definitions in Fig. 7) as a function of $k_{y}$ and $k_{x}$, respectively, for oblique modes in the PRB flow with magnetic field. Thin lines correspond to $\operatorname{Re}_{I, 1}-\operatorname{Re}_{L}$ and heavy lines to $\operatorname{Re}_{I, 2}-\operatorname{Re}_{L}$. The plots are given for three values of $\mathrm{Ha}$ : $\mathrm{Ha}=0.5$ (solid curves), $\mathrm{Ha}=1$ (dashed curves), and $\mathrm{Ha}=5$ (long-and-shortdashed curves). Note that the values plotted have been increased by a factor 500 for $\mathrm{Ha}=0.5$ and by a factor 150 for $\mathrm{Ha}=1$. The results are obtained for $\operatorname{Pr}=0.001$.

quence, the PRB flow subjected to a spanwise magnetic field can only be perturbed by transverse $(T)$ or longitudinal $(L)$ modes. This confirms the relevance of our previous discussion focused on the $(T)$ and $(L)$ modes (Secs. III A-III C).

\section{ENERGY ANALYSIS}

The kinetic energy budget associated with the transverse $\left(T_{1}\right)$ modes at critical values of Ra has been computed for the case $\operatorname{Pr}=0.01$ for increasing values of Re and different Hartmann numbers. Note that the transverse modes correspond to two-dimensional instabilities which develop in the $(x, z)$ plane, with no dependence on the $y$ direction and $v=0$. The variation with $\mathrm{Re}$ of the different energy terms is shown in Figs. 9 and 10. We see in Fig. 9 that the magnetic energy term $E_{m}$ remains zero, independently of $\mathrm{Re}$, which is consistent with the fact that the spanwise magnetic field has no effect on these transverse instabilities. In fact, we see that there is a perfect balance between the magnetic energy term connected to the directly induced current $E_{m, B}$ and the magnetic energy term resulting from the electric potential $E_{m, \phi}$. It can be shown as a general result ${ }^{16}$ that the term $E_{m, B}$ is al-

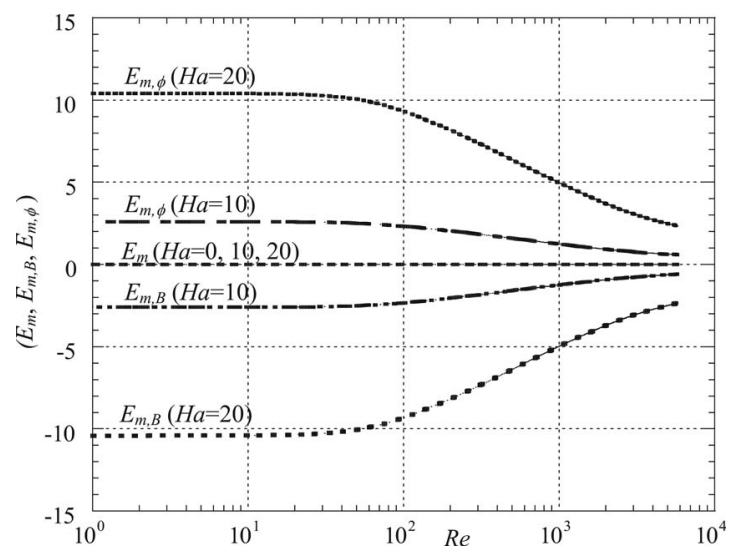

FIG. 9. Variation in the magnetic energy term $E_{m}$ and its contributions $E_{m, B}$ and $E_{m, \phi}$ as a function of the Reynolds number Re for the $\left(T_{1}\right)$ modes in the $\mathrm{PRB}$ flow, for different values of $\mathrm{Ha}$ and $\mathrm{Pr}=0.01$. 


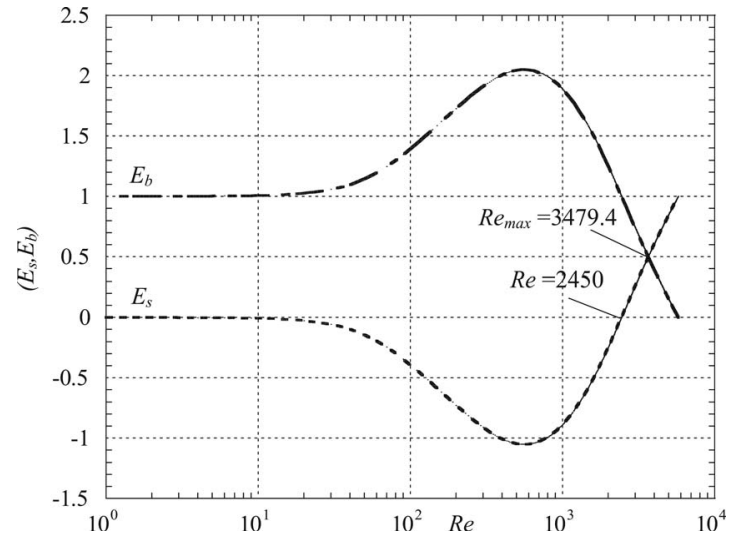

FIG. 10. Variation in the shear energy term $E_{s}$ and buoyancy energy term $E_{b}$ as a function of the Reynolds number Re for the $\left(T_{1}\right)$ modes in the PRB flow at $\operatorname{Pr}=0.01$. These energy terms do not depend on the Hartmann number.

ways stabilizing $\left(E_{m, B} \leq 0\right)$, the term $E_{m, \phi}$ always destabilizing $\left(E_{m, \phi} \geq 0\right)$, and the global term $E_{m}$ always stabilizing $\left(E_{m} \leq 0\right)$. In our case, the destabilizing contribution $E_{m, \phi}$ completely equilibrates the stabilizing contribution $E_{m, B}$ to give $E_{m}=0$. These two contributions decrease in intensity when Re is increased, in connection with the changes that affect the thermally induced instability when the shear flow is applied. The change in the instability with Re can be better understood through the variations in the buoyancy $E_{b}$ and shear $E_{s}$ energy terms given in Fig. 10. For $\operatorname{Re}=0$, the instability is thermally induced by buoyancy $\left(E_{b}=1\right.$ and $\left.E_{s}=0\right)$. We see that for moderate Reynolds numbers $(0 \leq \operatorname{Re} \leq 2450)$, the shear term has a stabilizing influence $\left(E_{s}<0\right)$, which induces an increase in the destabilizing buoyancy term in order to retain the energy balance necessary to trigger the instability. Beyond $\operatorname{Re}=2450$, however, the shear energy term becomes positive so that both $E_{s}$ and $E_{b}$ energy terms are now destabilizing, the influence of $E_{s}\left(E_{b}\right)$ increasing (decreasing) with increasing Re. Note that the maximum value of the critical Rayleigh number for these $\left(T_{1}\right)$ modes (Fig. 4 and Table II) is reached for equal $E_{s}$ and $E_{b}$ contributions $\left(E_{s}=E_{b}=0.5\right)$ at $\operatorname{Re}=3479.4$. With a further increase in $\mathrm{Re}$, the shear energy term $E_{s}$ becomes dominant, until $\operatorname{Re}_{0}$ where the instability becomes a pure shear instability $\left(E_{s}=1\right.$ and $\left.E_{b}=0\right)$ corresponding to the Tollmien-Schlichting waves. Note finally that, as expected, the variations with $\operatorname{Re}$ of the $E_{s}$ and $E_{b}$ energy terms are not affected by the spanwise magnetic field.

The kinetic energy budget associated with the longitudinal $(L)$ modes at critical values of $\mathrm{Ra}$ has also been computed for the case $\operatorname{Pr}=0.01$ for increasing values of $\operatorname{Re}$ and different Hartmann numbers. These longitudinal modes with $k_{x}=0$ correspond to instabilities that develop in the $(y, z)$ plane, with no dependence on the $x$ direction, but the $u$ velocity, forced by the term $-D U_{0}(z) w$ [see Eq. (17)], is not zero. A quick look at Eqs. (16)-(21) (considered for $k_{x}=0$ ) shows that $v, w, \theta$, and $p$ are coupled together inside Eqs. (16) and (18)-(20) (denoted as RB system), whereas they are independent of $U_{0}, u$, and $\phi$. In contrast, $u$ and $\phi$ are coupled together inside Eqs. (17) and (21) (denoted as S system) and depend on $U_{0}$ and $w$. The critical values of $\operatorname{Ra}$ for the $(L)$ modes are in fact only connected to the RB system, which does not depend on the basic shear flow and depends on the magnetic field only through the directly induced current. As a consequence, it is interesting to write separately the kinetic energy budget related to the velocity equations in the $(y, z)$ plane (equations in the RB system) and the kinetic energy budget related to the equation along the $x$ direction (equation in the S system). Each budget is normalized by its own viscous dissipation term, $K_{d,(y, z)}$ for the velocity equations in the $(y, z)$ plane and $K_{d, x}$ for the velocity equation in the $x$ direction. The results for these two kinetic energy budgets are given in Table IV. Both of these budgets have been found independent of Re, in contrast with the global budget expressed by Eq. (27) which would give a Re dependence. In the $(y, z)$ plane, because the equations are independent of the basic shear flow and of the electric potential, the budget will be reduced to $E_{b}+E_{m, B}=1$. There is obviously no dependence with $\operatorname{Re}$ in this case, and the stabilizing influence of the magnetic term $E_{m, B}$ will increase with $\mathrm{Ha}$ and induce an increase in the destabilizing buoyancy energy term $E_{b}$ to retain the energy balance. In the $x$ direction, in contrast, the budget will depend on the basic shear flow but not on buoyancy. For $\operatorname{Re}>0$, this budget can be written as $E_{s}+E_{m, B}$ $+E_{m, \phi}=1$. Despite the dependence on the basic shear flow, this budget is still found independent of Re. This is due to the normalization by $K_{d, x}$. In fact, before normalization, all the terms in this budget have a same dependence with Re, and this dependence disappears after normalization. To illus-

TABLE IV. Energy budgets in the $(y, z)$ plane and in the $x$ direction for the $(L)$ modes in the PRB flow at $\operatorname{Pr}=0.01$ (different values of $\mathrm{Ha}$ and varying $\mathrm{Re}$ ). Each budget is normalized by its own viscous dissipation term, $K_{d,(y, z)}$ and $K_{d, x}$, respectively. The terms of buoyancy energy $E_{b}$ and magnetic energy $E_{m, B}$ are involved in the first budget; the terms of shear energy $E_{s}$ and magnetic energy $E_{m, B}$ and $E_{m, \phi}$ are involved in the second budget. Both budgets are found independent of Re. The ratio between the two viscous dissipation terms, $R_{d}=K_{d, x} / K_{d,(y, z)}$, however, increases as $\mathrm{Re}^{2}$.

\begin{tabular}{|c|c|c|c|c|c|c|c|}
\hline \multirow[b]{2}{*}{$\mathrm{Ha}$} & \multicolumn{2}{|c|}{$(y, z)$ plane } & \multicolumn{4}{|c|}{$x$ direction } & \multirow[b]{2}{*}{$R_{d}$} \\
\hline & $E_{b}$ & $E_{m, B}$ & $E_{s}$ & $E_{m, B}$ & $E_{m, \phi}$ & $E_{m}$ & \\
\hline 0 & 1 & 0 & 1 & 0 & 0 & 0 & $13.643 \times 10^{-4} \mathrm{Re}^{2}$ \\
\hline 10 & 1.863 & -0.863 & 1.283 & -2.189 & 1.906 & -0.283 & $6.628 \times 10^{-4} \mathrm{Re}^{2}$ \\
\hline 20 & 3.257 & -2.257 & 1.724 & -9.222 & 8.498 & -0.724 & $2.549 \times 10^{-4} \mathrm{Re}^{2}$ \\
\hline
\end{tabular}


trate that, we have calculated $R_{d}$ the ratio between the two viscous dissipation terms, $R_{d}=K_{d, x} / K_{d,(y, z)}$ (Table IV). $R_{d}$ increases with $\operatorname{Re}$ as $\operatorname{Re}^{2}$, with a constant prefactor that can be exactly determined and decreases as $\mathrm{Ha}$ is increased. This $\mathrm{Re}^{2}$ dependence is connected to the following relationships between the perturbations: $u / v \sim u / w \sim u / \theta \sim \operatorname{Re}$. Concerning the budget in the $x$ direction, the shear energy term $E_{s}$ balances the viscous dissipation and the magnetic energy term $E_{m}$. Note that this $E_{m}$ term remains smaller than in the $(y, z)$ plane, but it is obtained from large $E_{m, B}$ and $\left|E_{m, \phi}\right|$ contributions (more than ten times larger than $E_{m}$ for $\mathrm{Ha}=20$ ). We have to recall that what occurs in the $x$ direction does not influence the values of $\mathrm{Ra}_{c}$ and the type of modes that become unstable. The budget in the $x$ direction then only reflects what is induced in this direction by the critical modes and the shear flow.

\section{CONCLUSION}

The magnetic field is usually used to delay the appearance of instabilities in electrically conducting fluid flows. ${ }^{11,16}$ In the present work, we have shown that besides this stabilizing effect, the magnetic field can be responsible for a selective control of the instabilities. In the case where the flow is subject to more than one single unstable mode, the magnetic field may have a different effect on these modes and make one of them become dominant.

For the PRB flow, our study has highlighted that a judiciously oriented magnetic field can be used to operate such a selective control. It was well known that without magnetic field $(\mathrm{Ha}=0)$, the three-dimensional longitudinal stationary modes were the dominant modes over a large range of Reynolds numbers $(0<\operatorname{Re} \leq 5490)$, whereas the twodimensional transverse traveling modes were the dominant modes only in a narrow interval $(5490 \leq \operatorname{Re} \leq 5772)$ in the neighborhood of $\operatorname{Re}_{0}=5772$, the critical value of $\mathrm{Re}$ for the isothermal Poiseuille flow. In contrast, our numerical calculations have shown that a spanwise magnetic field makes the transverse traveling modes prevail over large ranges of Reynolds number, for small Reynolds numbers $(0<\mathrm{Re}$ $\left.\leq \operatorname{Re}_{L}\right)$ as well as for large Reynolds numbers $\left(\operatorname{Re}_{R}<\operatorname{Re}\right.$ $\leq \mathrm{Re}_{0}$ ). The extent of these Reynolds number ranges is found to increase with increasing Hartmann number. Moreover, when the Hartmann number exceeds a limiting value $\mathrm{Ha}_{\text {lim }}$, the transverse traveling modes are the only thermally induced modes that affect the PRB flow. Note that this change in the dominant modes with Ha depends on the Prandtl number and is slower for larger Pr values. This induces an increase in $\mathrm{Ha}_{\text {lim }}$ with $\mathrm{Pr}$, which can be scaled as $\mathrm{Ha}_{\lim } \sim \mathrm{Pr}^{0.5}$. It has been finally checked that the oblique modes (with arbitrary orientations of the wave vector) never become the dominant modes when the spanwise magnetic field is applied.

Such selective controls could be of a crucial importance from a technological and industrial point of view. By allowing the enhancement of both heat and mass transfers because of the oscillatory character of the transverse modes, the selective control presented in this study should contribute to optimize the processes in which a PRB flow is involved.

${ }^{1}$ K. F. Jensen, E. O. Einset, and D. I. Fotiadis, "Flow phenomena in chemical vapor deposition of thin films," Annu. Rev. Fluid Mech. 23, 197 (1991).

${ }^{2}$ N. K. Ingle and T. J. Mountziaris, "The onset of transverse recirculations during flow of gases in horizontal ducts with differentially heated lower walls," J. Fluid Mech. 277, 249 (1994).

${ }^{3}$ M. Hasnaoui, E. Bilgen, P. Vasseur, and L. Robillard, "Mixed convective heat transfer in a horizontal channel heated periodically from below," Numer. Heat Transfer, Part A 20, 297 (1991).

${ }^{4}$ E. Bilgen, X. Wang, P. Vasseur, F. Meng, and L. Robillard, "On the periodic conditions to simulate mixed convection heat transfer in horizontal channels," Numer. Heat Transfer, Part A 27, 461 (1995).

${ }^{5}$ A. Tangborn, "A two-dimensional instability in a mixed convection flow with spatially periodic temperature boundary conditions," Phys. Fluids A 4, 1583 (1992).

${ }^{6} \mathrm{~S}$. Q. Zhang and A. V. Tangborn, "Flow regimes in two-dimensional mixed convection with spatially periodic lower wall heating," Phys. Fluids 6, 3285 (1994).

${ }^{7}$ H. K. Moffat and K. F. Jensen, "Complex flow phenomena in MOCVD reactors-1. Horizontal reactors," J. Cryst. Growth 77, 108 (1986).

${ }^{8}$ H. K. Moffat and K. F. Jensen, "Three-dimensional flow effects in silicon CVD in horizontal reactors," J. Electrochem. Soc. 135, 459 (1988).

${ }^{9}$ J. Ouazzani and F. Rosenberger, "Three-dimensional modelling of horizontal chemical vapor deposition-1. MOCVD at atmospheric pressure," J. Cryst. Growth 100, 545 (1990).

${ }^{10}$ C. R. Kleijn and C. J. Hoogendoorn, "A study of 2-D and 3-D transport phenomena in horizontal chemical vapor deposition reactors," Chem. Eng. Sci. 46, 321 (1991).

${ }^{11}$ W. Fakhfakh, S. Kaddeche, A. Abdennadher, D. Henry, and H. Ben Hadid, "Stability of the Hartmann flow heated from below," C. R. Mec. 334, 332 (2006).

${ }^{12} \mathrm{G}$. Evans and R. Greif, "A study of traveling wave instabilities in a horizontal channel flow with applications to chemical vapor deposition," Int. J. Heat Mass Transfer 32, 895 (1989).

${ }^{13}$ G. Evans and R. Greif, "Unsteady three-dimensional mixed convection in a heated horizontal channel with applications to chemical vapor deposition," Int. J. Heat Mass Transfer 34, 2039 (1991).

${ }^{14}$ A. V. Tangborn, S. Q. Zhang, and V. Lakshminarayanan, "A threedimensional instability in mixed convection with streamwise periodic heating," Phys. Fluids 7, 2648 (1995).

${ }^{15}$ S. Q. Zhang and A. V. Tangborn, "Two- and three-dimensional heat transfer in mixed convection with streamwise periodic heating," Numer. Heat Transfer, Part A 28, 431 (1995).

${ }^{16}$ S. Kaddeche, D. Henry, and H. Ben Hadid, "Magnetic stabilization of the buoyant convection between infinite horizontal walls with a horizontal temperature gradient," J. Fluid Mech. 480, 185 (2003).

${ }^{17}$ R. Moreau, Magnetohydrodynamics (Kluwer, Dordrecht, 1990).

${ }^{18} \mathrm{U}$. Burr and U. Müller, "Rayleigh-Bénard convection in liquid metal layers under the influence of a horizontal magnetic field," J. Fluid Mech. 453, 345 (2002).

${ }^{19}$ K. Fujimura and R. E. Kelly, "Interaction between longitudinal convection rolls and transverse waves in unstably stratified plane Poiseuille flow," Phys. Fluids 7, 68 (1995).

${ }^{20} \mathrm{~S}$. Chandrasekhar, Hydrodynamic and Hydromagnetic Stability (Dover, Oxford, 1961)

${ }^{21}$ H. Horiike, S. Konishi, H. Kondo, and A. Yamaguchi, "Liquid metal cooling issues for fusion and fission," Fusion Eng. Des. 83, 943 (2008).

${ }^{22}$ K. Fujimura and R. E. Kelly, "Degenerate bifurcation in stably stratified plane Poiseuille flow," J. Fluid Mech. 331, 261 (1997).

${ }^{23}$ K. S. Gage and W. H. Reid, "The stability of thermally stratified plane Poiseuille flow," J. Fluid Mech. 33, 21 (1968).

${ }^{24} \mathrm{Ch}$. Jung, M. Lücke, and P. Büchel, "Influence of through-flow on linear pattern formation properties in binary mixture convection," Phys. Rev. E 54, 1510 (1996).

${ }^{25}$ J. Hu, H. Ben Hadid, and D. Henry, "Linear stability analysis of Poiseuille-Rayleigh-Bénard flows in binary fluids with Soret effect," Phys. Fluids 19, 034101 (2007). 